\title{
ViSA-EEG: A user-centered application for visualization and statistical analysis of EEG signals
}

\author{
Yoselyn Nohemí Ortega-Gijón, Carmen Mezura-Godoy
}

Published: 30 November 2020

\begin{abstract}
The most common applications for human EEG research are: Neuromarketing, Human factors, Social interaction, Psychology and Neuroscience, Clinical and Psychiatric Studies and Brain Computer Interfaces (BCI). In the BCI research, the existence of software for the analysis of EEG signals is observed, however its functionality is limited to showing the signals and certain predefined characteristics, almost focused on the medical area, allowing a visual analysis by an expert, in addition to the fact that this software is developed depending on the signals to be analyzed and on the BCI itself. Therefore, for the development of BCIs, it is necessary for the developer to have software that allows him to visualize and analyze the EGG signals, in addition to being able to test and compare the interaction of users through the BCI, as well as his brain activity during the interaction and being able to observe the causes. In this context, we propose ViSA-EEG web application that allows BCI developers to visualize and analyze the EEG signals. This application was developed under a user-centered approach, involving the user in each of the four development phases and using different UX tools and techniques.
\end{abstract}

\section{Keywords}

User centered design, Usability, User eXperience - UX, Electroencephalography-EEG, Brain Computer Interface-BCI.

\section{Introduction}

The Human-Computer Interaction- IHC studies how people interact with computers and to what extent computers develop to improve interaction with people, its study components are: humans, computers and the interaction between them [9].

Human interaction with the computer involves three processes. The first comprises the cognitive functions performed when the human being receives, interprets and stores information, at the moment of sensory perception, then an action is performed in

Ortega-Gijón, Y.N., Mezura-Godoy, C.

Facultad de Estadística e Informática

Universidad Veracruzana

Xalapa, Veracruz, México.

zS18016065@estudiantes.uv.mx,cmezura@uv.mx accordance with previous perception. The second refers to the interaction, when the action performed by the human is sent to an input interface, at that moment the recognition of the action is performed, the representation and ends with the sending to the output interface, to start again the cycle with perception. The third involves the recognition of the actions by the computer and the subsequent representation, the computer is responsible for interpreting the user's cognitive functions and executing the actions, giving the user feedback to continue the cycle.

Figure 1 shows the architecture of a Graphical User Interface GUI, where the 3 important parts of the Human-Computer Interaction are located.

Particularly there is an interest in the development of applications with Brain Computer Interfaces-BCI. The term interface is used to name the functional connection that exists between two programs, systems or devices, which provides communication at various levels, making possible an exchange of information



Figure 1. Architecture of the Graphical User Interfaces

In BCI, this exchange of information takes place between the electrical activity of the brain and the device to be controlled [8]. The EEG measures the electrical activity of the brain at different sites in the head, generally using electrodes placed on the scalp. In the specific case of the BCI, mainly due to their architecture and nature, the traditional evaluation methods such as interviews and questionnaires are not necessary the best methods, because they present the following problems: they do not give information in real time, an information bias may arise or influence the evaluation, since the user may be tired after the interaction with BCI. So, the use of EEG has been proposed to evaluate the usability of BCIs during user interaction $[7,16]$.

There are different works that use algorithms for the analysis of EEG signals $[1,2,3,7,10,11,13,17,18]$, as well as different 
software or applications that perform the analysis $[4,6,14,19]$. But in these works, the algorithms used were coded specifically for each case study and in the case of the existing software, the license is too expensive, and its functionality is limited to showing the signals and certain predefined characteristics, almost they are focused on the medical area, allowing a visual analysis only by an expert, also they are mostly software desktop.

Therefore, for the development of BCIs, developers need software that allows them to visualize and analyze the EGG signals, in addition to test and to compare the interaction of users with the application through the BCI, as well as his brain activity during the interaction and being able to observe the causes. The present work focuses on the development of ViSA-EEG, a web application that allows: 1) data acquisition, 2) visualization of the EEG signals and 3) statistical analysis of the EEG signals. For statistical analysis, fast Fourier transform algorithms were used to obtain the frequency spectrum and wavelets (Daubechies 8) to obtain the frequency bands. This application was developed under a user-centered approach, involving the user in each of the four development phases and using different UX tools and techniques.

The article is organized as follows: in section 2 the usercentered design approach is presented, the same as the development of ViSA-EEG, in sections 3, 4, 5 and 6 the analysis and design of the application and finally in section 7 the conclusions and future work are presented.

\section{User-centered design process}

User-centered design is based on an explicit understanding of users, tasks, and environment. Users are involved in the entire design process. The user centered design has four phases according to ISO 9241-210: 2010 [5]:

- Understand and specify the context of use: To define it, it is necessary to incorporate a methodology that includes central user research.

- $\quad$ Specify usage requirements: which are derived from the tasks that the user performs in a context scenario.

- Develop a design solution that meets the use requirements: Based on the use requirements, a design solution is launched to verify them, and a low or high-fidelity prototype will be made depending on what is necessary for the test.

- Evaluate the design solution with the usage requirements: Finally, the solution is tested with real users that meet the user's characteristics and a usability test is done, to correct possible deficiencies, and re-iterate the process.

Figure 2 shows the phases of the development process with human-centered design, observing the order of each phase and the correct iteration between them.

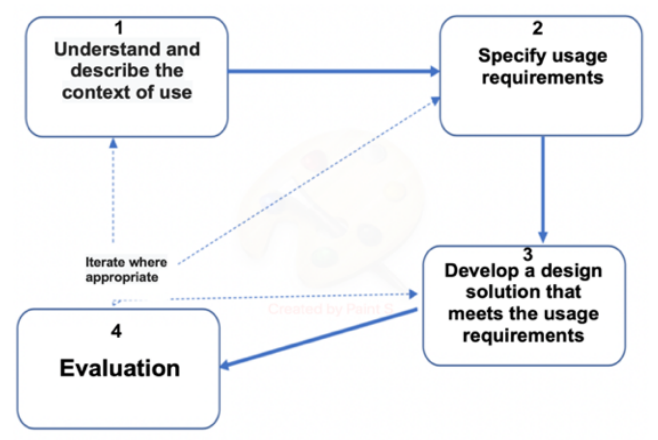

Figure 2. Graph with the 4 phases of the ISO 9241 process
Ten people participated in the Visa design process ( 4 men and 6 women between 22 and 30 years old), all of them with experience in software development. For each design phase, various UX techniques were used that allowed feedback from the participants.

\section{Understand and describe the context of use}

The context of use is determined by: The characteristics of the user, the most common tasks, equipment, the social and physical environment where the equipment is used.

\subsection{Characteristics of the User}

Once our target audience is identified, it is necessary to empathize, understand them as people in a context, who have needs, motivations, expectations and aspirations that we must understand.

The empathy map helped humanize end-users and empathize with them so that decisions are made based on their needs and expectations, which have become a common goal. Empathy Mapping is simplified into 5 main categories: 1) think / feel, 2) see, 3) hear, 4) say / do, 5) needs and pain, and focus on one person within a specific situation relevant to the domain of the product.

The profile of the user to whom the product is directed is to a BCI developer, whose main need is to make evaluation of BCI interfaces with veracity in the information to improve the quality of their work

This type of user is characterized according to the 5 categories by: 1) think/feel: about the architecture of the interfaces, the loss of information, the bad interaction with clients and poor performance, 2) see: problems in hardware and software, irresponsible people, the demand and the work overload, 3) hears: I don't have time, responsibility, 4) say/do: simultaneous tasks, be tired, evaluation tests constantly, waste of time, improve interaction and endless working hours, 5) needs: information overload, time insufficient.

To create the empathy map, a focus group was held where users participated.

Figure 3 presents the empathy map developed, presenting as main needs to improve its Interfaces through usability Assessment, minimize time in testing and evaluation, and improve the quality of its work.



Figure 3. Empathy map of a BCI developer

\subsection{Context of Use}

In a focus group session, users answered the following questions: What Characteristics should the application have?. The most 
common responses were: easy access, security, cross-platform, limited hard disk space. Analyzing the recovered information, it is observed that a viable option was to build a web application, since it could be executed with few computer resources and without the need for prior installation.

\subsection{Equipment}

The information retrieved from the users in the focus group session could establish the development of a web application, with the following equipment specifications: i) System requirements: SO Windows (7,8 y 10) 64, Ubuntu, MacOs, ii) Platform: Web and iii) Preferably browsers: Chrome and Safari.

\section{Specify usage requirements}

To obtain requirements and to visualize user's experience as they interact with the application the Customer Journey Map tool was used. The Customer Journey Map describes the user's interaction points and helps to visualize the main functionalities. A focus group was also carried out where the users expressed the main needs to be solved.

-Requirements: BCI developers are the main users of ViSA-EEG. These users need to analyze EEG signals, in order to for create new BCI or improve them. This application must allow users to visualize the signals obtained in your tests, compare the interaction of the participant with their brain activity during the interaction, observe the behavior of the signals through the spectrum and frequency bands, being able to visually detect any sudden change during the interaction and look for the causes of it. The list of requirements was: 1) easy and friendly access, 2) to show all channels properly, separating each one, 3) to observe the entire length of the signal, 4) to visualize the bands and spectra, 5) to visualize the user during the interaction for analysis, 6) to improve analysis time and 7) to make statistical analysis.

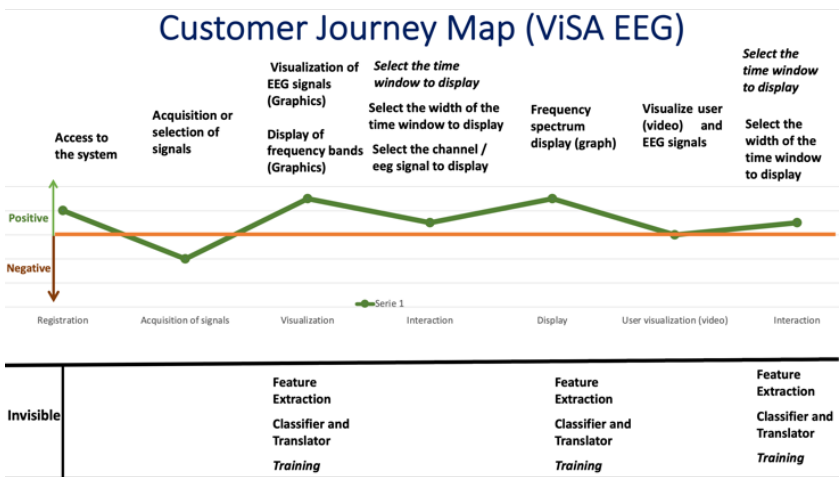

Figure 4. Customer Journey Map.

-User's experience: The points of interaction were analyzed, starting with access to the system and ending with the user and the signs. For each point of interaction, the user experience was evaluated based on the requirements and needs, analyzing the scope that the application should have. In Figure 4 the Customer Journey Map is presented. The green line presents de positive o negative experience.

\section{Development of design solution}

Once the user requirements have been defined, we proceed to the design phase, by designing low and high-fidelity prototypes. To carry out this phase, design tools are used, particularly were used in Sketch, Wireframes and Mockups. It began developing the Sketch which is a first paper sketch of the interface, then it continued with the Wireframes where there is a real visualization of the size and distribution of the controls, finally the Mockups where the basic functionalities of the system can already be appreciated like changing screens or displaying controls.

\subsection{Low Fidelity Prototypes}

At the beginning the Sketch tool was used was for the ViSA-EEG software. The first strokes on a sheet of paper was made. In the Sketch, the most characteristic elements such as logos, use of metaphors are defined. Also, the form of navigation and content and functional areas.

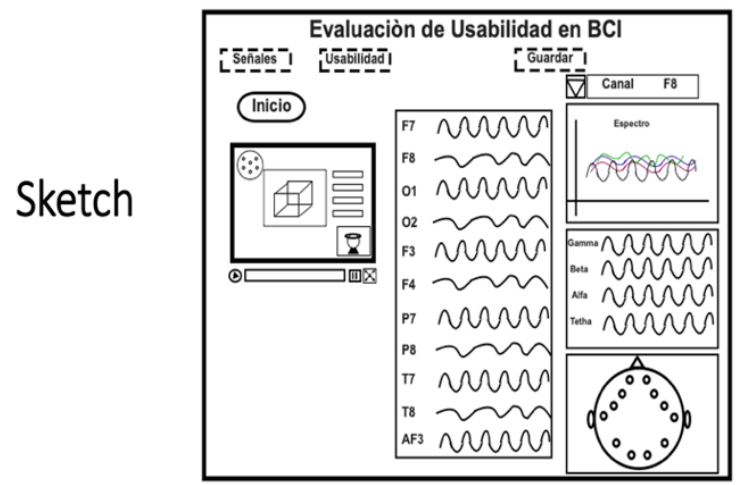

Figure 5. Sketch of the $1^{\text {st }}$ screen

Figure 5 shows the final sketch of the first screen that was built from the requirements, showing the distribution of the signal elements, bands and spectrum. The distribution of the components that were raised at the beginning was defined as follows: On the left side, the test user is displayed during the interaction with the BCI to be analyzed. In the central part are the EEG signals per channel. On the right side, the frequency spectrum, frequency bands, and busy montage are observed. With this structure user can visualize the test and the signals on time at the same time, as well as their bands and the spectrum of the signal to improve the analysis.

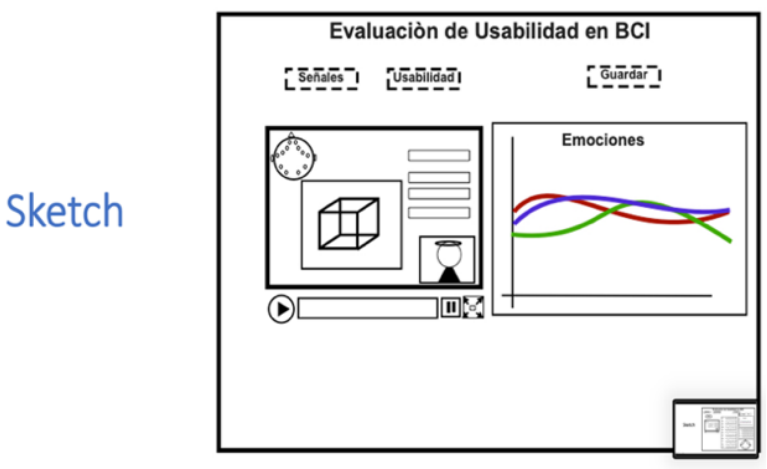

Figure 6. Sketch of the 2nd screen. 
Figure 6 shows the final sketch of the second screen that was built from the requirements, showing the location of the video and the EEG signals. The distribution of the components that were initially raised for the second screen was stipulated as follows: on the left side, the test user is shown during the interaction with the BCI to be analyzed. On the right side, the obtained EEG signals are observed. This distribution was based on the user's requirement to visualize the interaction and the EEG signals at the same time to be able to compare. Users participated by evaluating the design elements and providing feedback.

Later the Wireframe tool was used. The Wireframe connects the conceptual structure, or information architecture, with the visual design of the web or application. They help establish functionality, and relationships between different screen templates.

A Wireframe was developed for this application, through this process the hierarchy of the design information could be defined, and it was easier to plan the design according to how the user wants to process the information.

Figure 7 shows the Wireframe of the first screen that was built from the Sketch previously presented, showing the distribution of the elements of the signals, bands and spectrum. Again, the distribution of the first screen was proposed: on the left side, the test user is displayed during the interaction with the BCI to be evaluated. In the central part are the EEG signals per channel. On the right side, the frequency spectrum, frequency bands, and busy montage are observed.

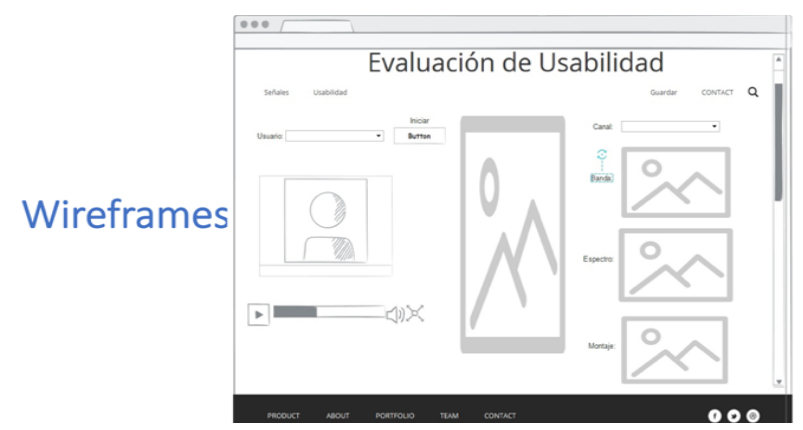

Figure 7. Wireframe of the 1 st screen.

Figure 8 shows the final Wireframe of the second screen that was built from the Sketch, showing the location of the video and synchronization with the signals. The same Sketch distribution of the second window was followed, displaying the user interaction on the left side and the EEG signals on the right side. With this wireframe it is still a complete design phase, giving a bigger picture of size and space. At this stage the components are still presented in black and white.

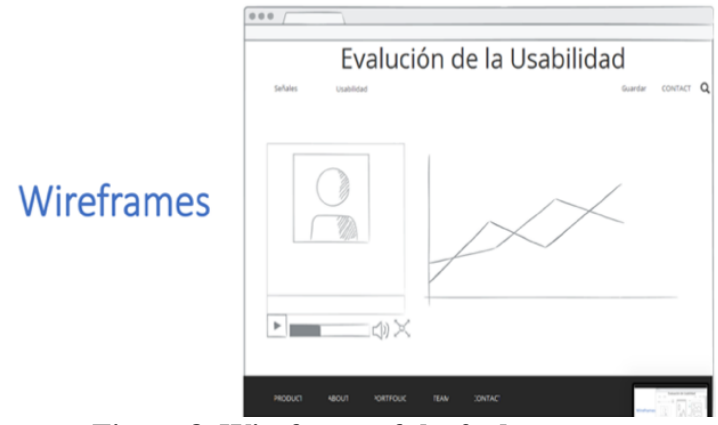

Figure 8. Wireframe of the 2nd screen
In the development and validation of the Wireframes, the users again had a strong participation. The Wireframes were shown to them on the screen, feedback was obtained, and changes were made, until the latest version was obtained. In particular, the size and location of the controls were validated, as well as the first functionalities were observed and verified. During Wireframe construction, users had better ideas of sizes and spaces and use of interface components.

\subsection{High Fidelity Prototypes}

For de development of the high-fidelity prototype Mockup tool was used. The Mockup is a means of representing the appearance of the product, it shows the fundamentals of its functionality. Mockups include visual details, such as colors, typography, etc., and are generally static. When looking at a mockup, you need to have a good idea of what the end product will look like and a rough idea of how it might work (even if the features haven't been developed yet). For the development of the Mockups more similar to what the application, web page, etc. will be, the ADOBE XD tool was used.

Figure 9 shows the Mockup of the first screen from the previous Wireframes, showing the elements, the colors to use and the graphic design. The distribution of the components is the same as that shown previously by the Sketch and Wireframe of the first screen, the main ones being the user's display, the EEG signals per channel, the spectrum, the frequency bands and the assembly. When making the Mockup, the idea of space and size of the components continued to be improved by occupying an image of real EEG signals, and the possible colors to be used in the application are also presented. For EEG signals each channel is represented with a color, in order to be able to locate and differentiate one from the other in the assembly.

The first functionalities are also shown, pointing out in the design the components that will perform some action by means of a small arrow, these components are the buttons that are at the top (Start, User, Bands).

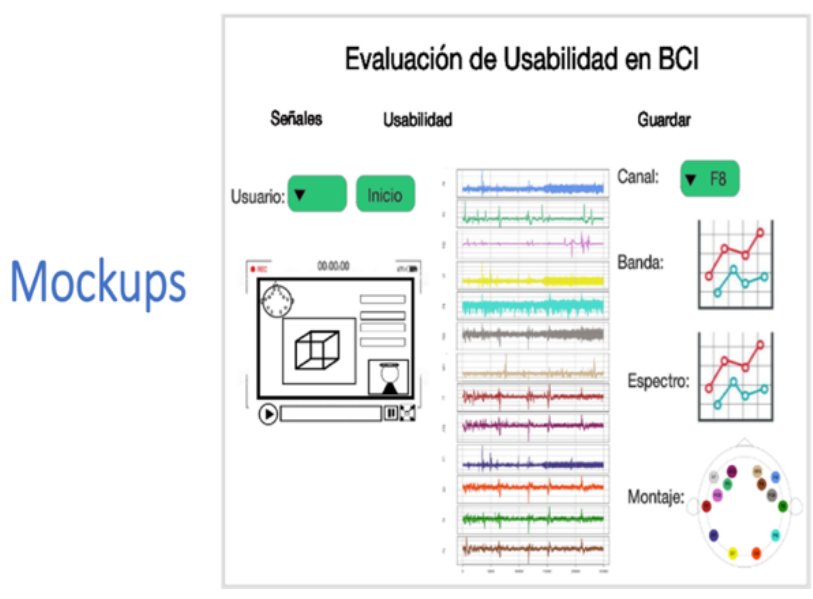

Figure 9. Mockup of the 1st screen

Figure 10 shows the Mockup of the second screen from the previous Wireframes and graphic design. The same distribution of the Sketch and the Wireframe is followed, but in this case the user's visualization during the interaction is represented with the BCI image of Emotiv, where the movement of a cube from concentration is performed. In the case of the signals, they are 
represented by the image of a graph and with the colors to be used in the final application.

\section{Evaluación de Usabilidad en $\mathrm{BCl}$}



Figure 10. Mockup of the 2nd screen.

In the operational part, the components that perform visualization functions such as change of graph, change of screen and modification of interactive graph are shown.

Figure 11 shows the Mockup, showing the transitions between the screens, giving greater functionality and graphic design.

\section{Mockups}



Figure 11. Mockup of the Interaction between the 1st and 2nd week

The mockups were validated by the users, the mockups were shown to them on the screen, feedback was obtained, and changes were made, until the latest version was obtained. In particular, the size, location of the controls and even the color of the interface, as well as the basic functionalities, were ratified. At this point user have a clear idea of how the final interface works.

\subsection{Implementation}

Once the interface design was completed and the functionalities were defined, the ViSA-EEG coding continued. The following describes: the implementation tools and the software architecture.

\subsubsection{Implementation tools}

The Shiny Package of RStudio was implemented, this package allows the construction of graphic Web interfaces and communication with Python, performing operations faster. The communication between Python and $\mathrm{R}$ is done through the "reticulate" package, from Shiny the function calls are made in Python and the result is received again.

Figure 12 details the programming languages, the development software and the packages or libraries used for the development of the application.

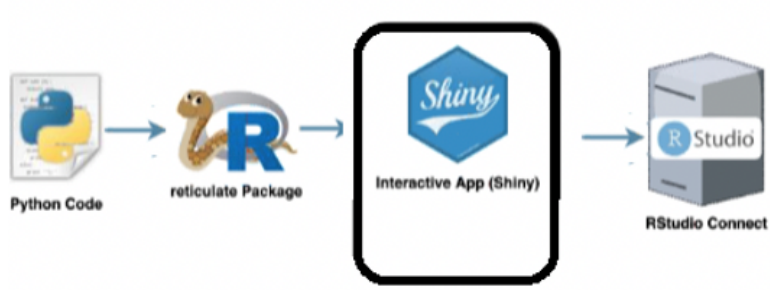

Figure 12. Implementation tools

\subsubsection{Software architecture}

The architecture of the application is as follows: 1) Graphic Interface: The user enters the web application and the start screen is displayed, where it proceeds to load the data of the EEG signals, sending a request to the server, then receives the response. 2) Server: Receives the request and performs the respective process by sending the result, the operations to be performed by the server are to read the EEG data, view the signals and perform the statistical analysis of the signals. Figure 13 presents the architecture of the application.

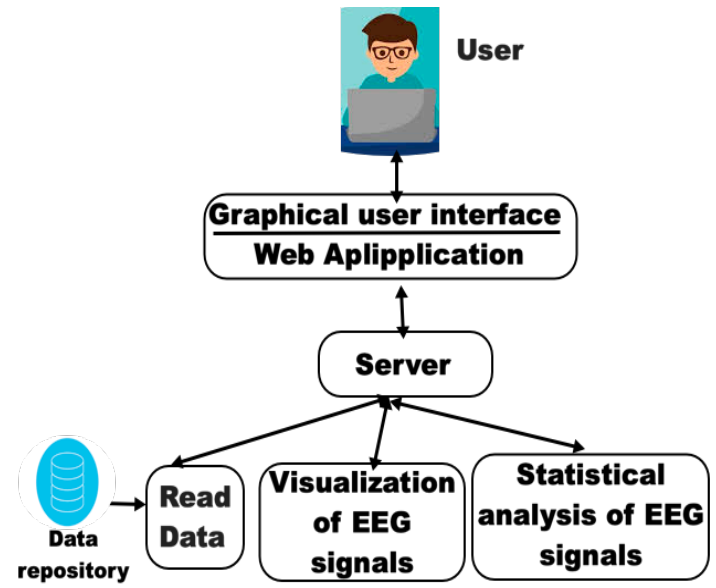

Figure 13. Components of software architecture

-Graphical User Interface: The interface of the web application is made up of the following elements: 1) Panels, which divide the sector of signals, bands and spectrum on the screen. 2) Tabs, to change the application section, between the signals section, the spectrum section and finally the user / video section. 3) Sidebar, where are the interaction controls to manipulate the signals. 4) Controls, there is the data load button, slider to select the window and the width of the window to be displayed, and finally a combo box to select the channel / signal.

-Data read: To read the signals, the file format is ".csv", and it must be structured as follows. Figure 14-left shows the structure of the ".csv" file, figure 13-right shows the location of the nodes. Each column represents a Channel, having a total of 14 channels (F3, F4, $\mathrm{F} 7, \mathrm{~F} 8, \mathrm{~T} 7, \mathrm{~T} 8, \mathrm{AF} 3, \mathrm{AF} 4, \mathrm{P} 7, \mathrm{P} 8, \mathrm{O} 1, \mathrm{O} 2, \mathrm{FC} 5, \mathrm{FC} 6)$, the rows are the records obtained from the channels. 


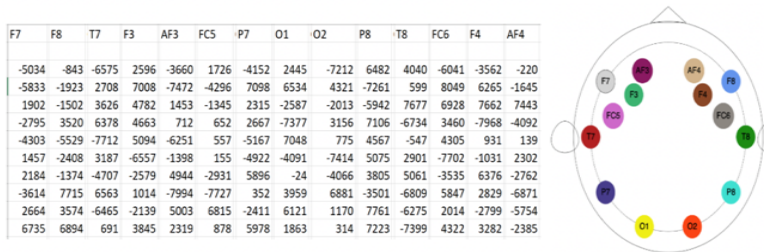

Figure 14. File specifications

- Statistical analysis of the EEG signals: The following algorithms were used for the statistical analysis of the signals: 1) Fast Fourier transform. - It is a mathematical transformation used to transform signals between the time (or spatial) domain and the frequency domain, which has many applications in physics and engineering, its applications are signal processing and in this work they deal with the acquisition of the frequency spectrum. 2) Waves. - It is a special type of mathematical transform that represents a signal in terms of translated and dilated versions of a finite wave (called the mother wave). Regarding its applications, the wave transform is used for signal encoding and analysis, being used in this work to obtain the frequency bands.

- Visualization of EEG signals: The button to load the "CSV" file is located in the upper left (See figure 15).

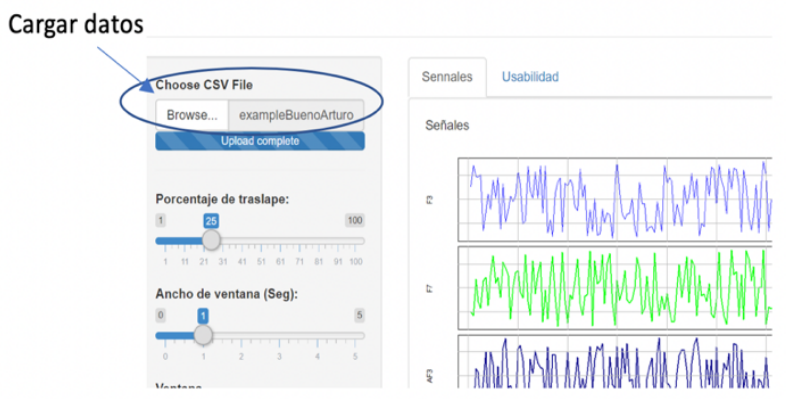

Figure 15. Data loading.

Once the signals are obtained, the EEG signals divided into channels can be viewed. These channels are the same as those presented in the assembly. At the moment of executing it, the following dialog window will appear to select said structured file with the specifications shown above.

Figure 16 shows the first second of time. of the signals. In order to display more, you can change the width of the window and the window to be displayed, changing the values on the left side of the menu. Regarding the frequency bands, the default "F3" channel bands are shown, to change it is also done through the menu.

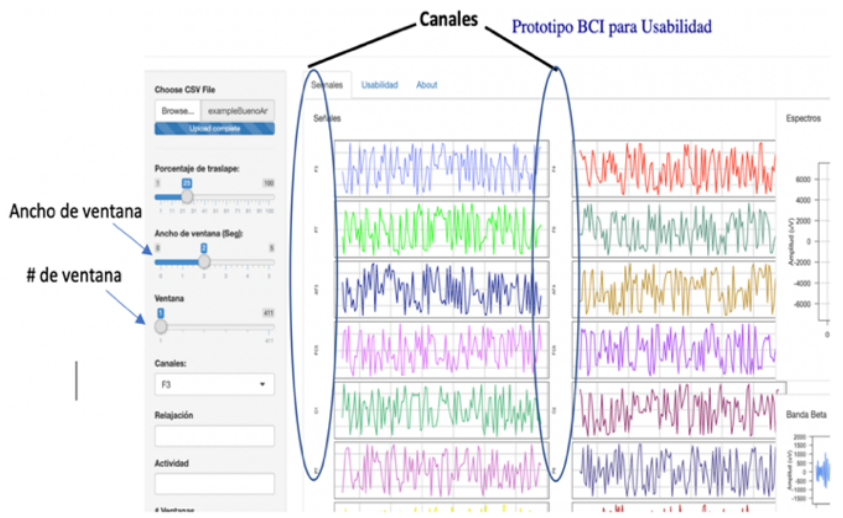

Figure 16. Visualization of EEG signals.

Once the signals have been obtained, the frequency bands and the frequency spectrum can be visualized, as shown in the following image. It is important to emphasize that when changing the width of the window and the \# of the window to show the EEG signals, it will also change the bands and the spectrum. Figure 17 presents the initial screen of the application and the location of the EEG signals, bands and frequency spectrum.

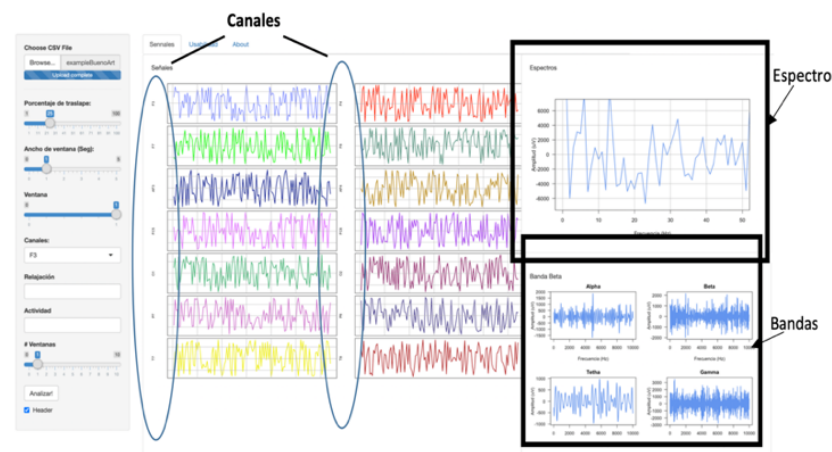

Figure 17. Visualization of EEG signals.

The distribution of the components is not the same as that previously proposed, given the size of the signals and the addition of a control panel (data load, time window, window size), it was necessary to change the distribution, adding a new window with user visualization and EEG signals.

Figure 18 shows the frequency spectra of the signals, with the functionality of changing the window width and the window to be displayed.

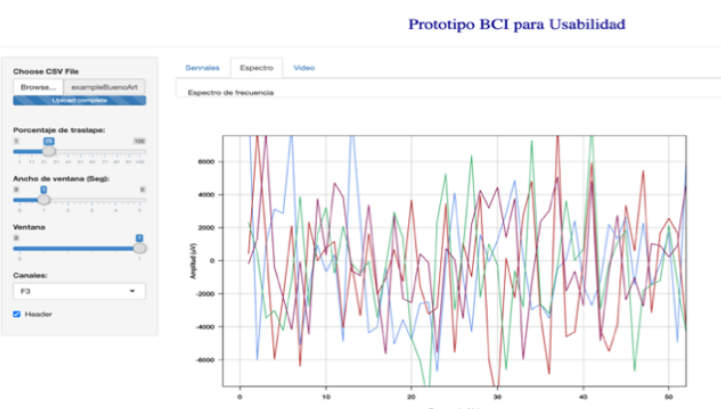

Figure 17. Visualization of the frequency spectrum. 
And finally, figure 19 of the video display and the EGG signals are shown, along with the frequency bands corresponding to the selected channel, located in the 3rd tab of the application.
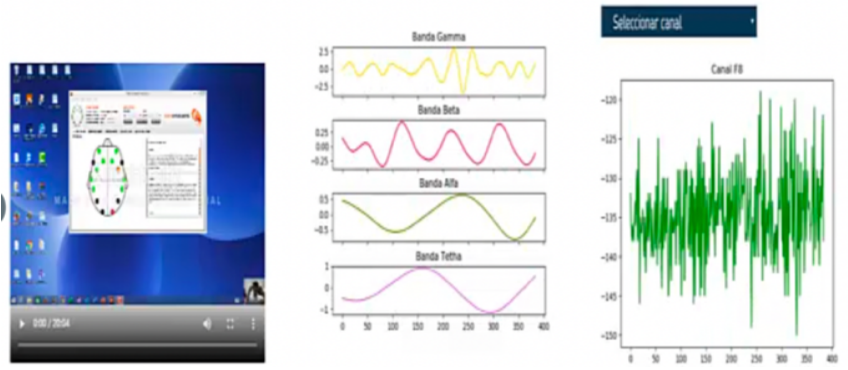

Figure 19. User visualization during the interaction.

\section{Evaluate the design solution}

For the evaluation it was necessary to define the heuristic evaluation method. There are different heuristics for testing / evaluation are called "heuristics", the most used heuristics are those of Jakob Nielsen, these are ten general principles for the design of the user interface [15]. Nielsen heuristics were used for the application tests, this evaluation was carried out by 5 experts in usability. Table 1 shows the characteristics of the evaluators such as age, sex and the level of experience in usability, where 1 represents the minimum and 5 the maximum level of experience.

Table 1. Characteristics of the evaluators.

\begin{tabular}{|c|c|c|}
\hline User & Sex & Usability experience \\
\hline 1 & Women & 5 \\
\hline 2 & Man & 3 \\
\hline 3 & Women & 4 \\
\hline 4 & Women & 5 \\
\hline 5 & Man & 4 \\
\hline
\end{tabular}

The experts proceeded to carry out the system tests, starting with the selection of the csv file to obtain and visualize the data, the visualization of the signals and ending with the functionality of the widgets.

Table 2 shows the results of the heuristic evaluation. Each evaluator proceeded to validate each heuristic in the application. A score of 1 to 5 was established, where 1 is the minimum value and 5 the maximum. It was also established that for elements evaluated on average with 3 or lower, they should be attended immediately and for elements evaluated between 5 and 4 on average they can be done later (the next version of the system). Work was done immediately on providing help and documentation and how to get out of fatal errors. It should be noted that in addition to the heuristic evaluation, the expert provided recommendations for improving the application.

In the results of the heuristic evaluation, the benefits of applying the human-centered design process can be observed, obtaining good results and with few observations to solve. Among the most important observations to improve the usability of the application, and that were contributed by the experts, are the following: 1) to include the level of interaction (know what is before and what is next), 2) to use iconography with metaphors for some functions and 3) provide help options. On the other hand, the positive points of the application are as follows: 1) minimalist design, 2) convenient interface colors, 3) proper design layout, and 4) consistent design.

Table 2. Table captions should be placed above the table.

\begin{tabular}{|c|c|c|c|c|c|c|}
\hline Heuristics & Description & 1 & $2 \mid 3$ & 34 & 45 & $\mathrm{R}$ \\
\hline $\begin{array}{l}\text { System state } \\
\text { visibility }\end{array}$ & $\begin{array}{l}\text { The system should always keep users } \\
\text { informed about what is happening, through } \\
\text { appropriate feedback in a reasonable time. }\end{array}$ & 5 & \begin{tabular}{l|l}
3 & 1
\end{tabular} & 15 & $5 \mid 4$ & 4 \\
\hline $\begin{array}{l}\text { Coincidence } \\
\text { between the } \\
\text { system and the } \\
\text { real world }\end{array}$ & $\begin{array}{c}\text { The system must speak in the language of the } \\
\text { user, with words, phrases and concepts } \\
\text { familiar to him. Use real-world conventions, } \\
\text { making information appear in a natural and } \\
\text { logical order. }\end{array}$ & 5 & \begin{tabular}{l|l}
5 & 4
\end{tabular} & & $5 \mid 4$ & 5 \\
\hline $\begin{array}{l}\text { User control } \\
\text { and freedom }\end{array}$ & $\begin{array}{l}\text { Users often choose features by mistake and } \\
\text { need an "emergency door" to get out of the } \\
\text { unwanted state. Offer support to undo and } \\
\text { redo actions. }\end{array}$ & 3 & 33 & 35 & 55 & 4 \\
\hline $\begin{array}{c}\text { Consistency } \\
\text { and standards }\end{array}$ & $\begin{array}{l}\text { Users should not have to wonder if the various } \\
\text { words, situations, or actions mean the same } \\
\text { thing. That the rules and conventions of the } \\
\text { platform on which the system is being } \\
\text { implemented are followed. }\end{array}$ & 3 & $5 \mid 5$ & 55 & $5 \mid 5$ & 5 \\
\hline $\begin{array}{c}\text { Error } \\
\text { prevention }\end{array}$ & $\begin{array}{l}\text { Rather than designing good error messages, it } \\
\text { is better to prevent the problem from occurring }\end{array}$ & 5 & & $5 \mid 5$ & $5 \mid 5$ & 5 \\
\hline $\begin{array}{l}\text { Recognition } \\
\text { better than } \\
\text { remember }\end{array}$ & $\begin{array}{l}\text { Minimize user memory load by making } \\
\text { objects, actions, and options visible. The user } \\
\text { should not have to remember information } \\
\text { from one part of the dialogue to another. }\end{array}$ & 3 & $5 \mid 5$ & $5 \mid 5$ & \begin{tabular}{l|l}
5 & 4
\end{tabular} & 4 \\
\hline $\begin{array}{c}\text { Flexibility and } \\
\text { efficiency of } \\
\text { use }\end{array}$ & $\begin{array}{c}\text { Accelerators, not seen by the beginning user, } \\
\text { improve interaction for the expert user in such } \\
\text { a way that the system can be used by } \\
\text { inexperienced and experienced users. It is } \\
\text { important that the system allows customizing } \\
\text { frequent actions. }\end{array}$ & 3 & & 35 & $5 \mid 5$ & 4 \\
\hline $\begin{array}{l}\text { Aesthetic and } \\
\text { minimalist } \\
\text { design }\end{array}$ & $\begin{array}{c}\text { Dialogues should not contain irrelevant or } \\
\text { rarely needed information. Each extra unit of } \\
\text { information in a dialogue competes with the } \\
\text { important information, decreasing its relative } \\
\text { visibility. }\end{array}$ & 5 & & $5 \mid 5$ & \begin{tabular}{l|l}
5 & 4
\end{tabular} & 5 \\
\hline $\begin{array}{c}\text { Help recognize, } \\
\text { diagnose and } \\
\text { recover from } \\
\text { errors }\end{array}$ & $\begin{array}{c}\text { Error messages should be expressed in plain } \\
\text { language (no codes), accurately indicating the } \\
\text { problem and suggesting a solution. }\end{array}$ & 1 & & $1 \mid 5$ & 53 & 3 \\
\hline $\begin{array}{c}\text { Help and } \\
\text { documentation }\end{array}$ & $\begin{array}{l}\text { Although it is better that the system can be } \\
\text { used without documentation, it is necessary to } \\
\text { provide the user with help and documentation. } \\
\text { This has to be easy to search, focused on the } \\
\text { user's tasks, with information on the stages to } \\
\text { be carried out and that is not very extensive. }\end{array}$ & 1 & & 35 & \begin{tabular}{l|l}
5 & 4
\end{tabular} & 3 \\
\hline
\end{tabular}

\section{Conclusion}

The user-centered design process aims to make systems usable and useful, by focusing on users, their needs and requirements, while applying ergonomic techniques and usability knowledge. This approach promotes effectiveness and efficiency, improves human well-being, user satisfaction, accessibility, and sustainability, taking into account the end user from conception and during development.

The phases and tools that were used in the design process are very important for development, since they helped make it clear to whom the application is directed, why its development is necessary, 
what are the requirements to satisfy, minimize time in the elaboration of the design at the moment of showing the users from the initial sketches to the final application, helping to make corrections from the beginning in a timely manner.

This application is aimed at all BCI developers, to analyze the EEG signals resulting from the interaction with their developed applications, having as objectives to visualize the signals completely and correctly, to be able to visualize the user and obtain a statistical analysis of the signals.

In the results of the heuristic evaluation, the benefits of applying the user-centered design process can be observed, obtaining good results and with few observations to resolve, such as helping to resolve errors and user help section. Given the results of the research, the line to follow is the analysis and tests for the measurement of different aspects / factors to contribute and improve the evaluation of usability in the BCI. Another aspect to cover more rigorous testing of the application with users and to update or add functionality.

\section{Acknowledgements}

This work was partially developed under the support of the National Council of Science and Technology (CONACYT) in the scope of the Catedras CONACYT project "Infraestructura para Agilizar el Desarrollo de Sistemas Centrados en el Usuario", Ref. 3053. In addition, we thank CONACYT for the doctoral scholarship number 785866 of the first author, as well as at Universidad Veracruzana for the support in the development of this research.

\section{References}

[1] Ansari-Asl, K., Chanel, G., and Pun, T. (2007). A channel selection method for EEG classification in emotion assessment based on synchronization likelihood. In 2007 15th European Signal Processing Conference (pp. 1241-1245). IEEE.

[2] Appriou, A., Cichocki, A., and Lotte, F. (2018). Towards robust neuroadaptive $\mathrm{HCI}$ : exploring modern machine learning methods to estimate mental workload from EEG signals. In Extended Abstracts of the 2018 CHI Conference on Human Factors in Computing Systems (pp. 1-6).

[3] Bhardwaj, A., Gupta, A., Jain, P., Rani, A., and Yadav, J. (2015). Classification of human emotions from EEG signals using SVM and LDA Classifiers. In 2015 2nd International Conference on Signal Processing and Integrated Networks (SPIN) (pp. 180-185). IEEE.

[4] Brunner, C., Andreoni, G., Bianchi, L., Blankertz, B., Breitwieser, C., Kanoh, S. I., ... and Perego, P. (2012). BCI software platforms. In Towards Practical Brain-Computer Interfaces (pp. 303-331). Springer, Berlin, Heidelberg.

[5] DIS, I. (2010). 9241-210: 2010. Ergonomics of human system interaction-Part 210: Human-centered design for interactive systems (formerly known as 13407). International Standardization Organization (ISO). Switzerland.
[6] Erkan, E., and Akbaba, M. (2018). A study on performance increasing in SSVEP based BCI application. Engineering Science and Technology, an International Journal, 21(3), 421-427.

[7] Frey, J., Pommereau, L., Lotte, F., and Hachet, M. (2014). Assessing the zone of comfort in stereoscopic displays using EEG. In CHI'14 Extended Abstracts on Human Factors in Computing Systems (pp. 2041-2046).

[8] Gentiletti, G., Tabernig, C., and Acevedo, R. (2007). Interfaz cerebro-computadora: Estado del arte y desarrollo en Argentina. Revista Argentina de Bioingeniería, Revista SABI, pp22-29, 13(1).

[9] Helander, M. G. (2014). Handbook of human-computer interaction. Elsevier.

[10] Hosseini, S. A., and Khalilzadeh, M. A. (2010). Emotional stress recognition system using EEG and psychophysiological signals: Using new labelling process of EEG signals in emotional stress state. In 2010 international conference on biomedical engineering and computer science (pp. 1-6). IEEE.

[11] Kumar, N., and Kumar, J. (2016). Measurement of cognitive load in HCI systems using EEG power spectrum: an experimental study. Procedia Computer Science, 84, 70-78.

[12] Kumar, J. (2016). Affective modelling of users in HCI using EEG. Procedia Computer Science, 84, 107-114.

[13] Liu, Y., Sourina, O., and Nguyen, M. K. (2010). Real-time EEG-based human emotion recognition and visualization. In 2010 international conference on cyberworlds (pp. 262269). IEEE.

[14] Nam, C. S., Nijholt, A., and Lotte, F. (2018). Braincomputer interfaces handbook: technological and theoretical advances. CRC Press.

[15] Jimenez, C., Lozada, P., and Rosas, P. (2016). Usability heuristics: A systematic review. In 2016 IEEE 11th Colombian Computing Conference (CCC) (pp. 1-8). IEEE.

[16] Ortega-Gijón, Y. N., and Mezura-Godoy, C. (2019). Usability evaluation process of brain computer interfaces: an experimental study. In Proceedings of the IX Latin American Conference on Human Computer Interaction (pp. 1-8).

[17] Parra, L. C., Spence, C. D., Gerson, A. D., and Sajda, P. (2003). Response error correction-a demonstration of improved human-machine performance using real-time EEG monitoring. IEEE transactions on neural systems and rehabilitation engineering, 11(2), 173-177.

[18] Wang, Q., Sourina, O., and Nguyen, M. K. (2010). Eeg-based" serious" games design for medical applications. In 2010 international conference on cyberworlds (pp. 270-276). IEEE.

Xing, X., Wang, Y., Pei, W., Guo, X., Liu, Z., Wang, F., ... and Chen, H. (2018). A high-speed SSVEP-based BCI using dry EEG electrodes. Scientific reports, 8(1), 1-10.

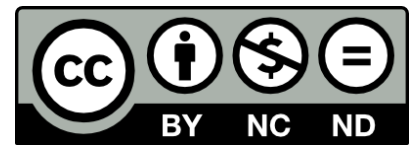

(C) 2020 by the authors. This work is licensed under the Creative Commons AttributionNonCommercial-NoDerivatives 4.0 International License. To view a copy of this license, visit http://creativecommons.org/licenses/by-nc-nd/4.0/ or send a letter to Creative Commons, PO Box 1866, Mountain View, CA 94042, USA. 